\title{
Structure of bottle-brush polymers in solution: A Monte Carlo test of models for the scattering function
}

\author{
Hsiao-Ping Hsu, Wolfgang Paul, and Kurt Binder \\ Institut für Physik, Johannes Gutenberg-Universität Mainz \\ D-55099 Mainz, Staudinger Weg 7, Germany
}

(Dated: October 25, 2018)

\begin{abstract}
Extensive Monte Carlo results are presented for the structure of a bottle-brush polymer under good solvent or theta solvent conditions. Varying the side chain length, backbone length, and the grafting density for a rigid straight backbone, both radial density profiles of monomers and side chain ends are obtained, as well as structure factors describing the scattering from a single side chain and from the total bottle-brush polymer. To describe the structure in the interior of a very long bottle-brush, a periodic boundary condition in the direction along the backbone is used, and to describe effects due to the finiteness of the backbone length, a second set of simulations with free ends of the backbone is performed. In the latter case, the inhomogeneity of the structure in the direction along the backbone is carefully investigated. We use these results to test various phenomenological models that have been proposed to interpret experimental scattering data for bottle-brush macromolecules. These models aim to extract information on the radial density profile of a bottle-brush from the total scattering via suitable convolution approximations. Limitations of this approach and the optimal way to perform the analysis of the scattering data within this approach are discussed.
\end{abstract}

\section{INTRODUCTION}

Recently there has been a great experimental (see e. g. 1, 2, 3, 4, 5, 6, 7]) and theoretical [8, 9, 10, 11, $12,13,14,15,16,17,18,19,20,21,22,23,24,25$, 26, 27, 28, 29, 30, 31, 32, 33, 34, 35] interest in the conformation of so-called bottle-brush polymers. Such polymers consist of a long (flexible) main chain, at which many flexible (shorter) side chains are densely grafted, such that an overall shape of a worm-like cylindrical brush results [1, 2, 3, 4, 5]. Synthesizing such polymers with suitable characteristics, materials can be prepared whose properties can be adjusted by external stimuli, such as the solvent quality, $\mathrm{pH}$, or temperature, and this fact makes such bottle-brush polymers interesting for various applications [36, 37]. For controlling the properties of such bottle-brush polymers, a good theoretical understanding of their structure and conformation, as a function of control parameters such as the chain lengths of the main chain and the side chains, their grafting density, and the solvent quality, is mandatory. However, despite the longstanding theoretical efforts $8,9,10,11,12,13,14,15,16,17,18,19,20,21$, $22,23,24,25,26,27,28,29,30,31,32,33,34,35]$ this problem is still incompletely understood. While one has various scaling predictions (see 34] for a recent review) and treatments based on the strong stretching limit of self-consistent field theory exist since a long time (e.g. Refs. [8, 9, 10, 11]), recent simulations 34] and also the experiments [3, 4, 5] indicate that the regime where these theories become accurate would require side chain lengths that are hardly accessible either by experiment or by simulation. As a consequence, the theoretical guidance for the interpretation of extensive experiments by combined light and small-angle neutron scattering analysis [3, 4, 5] is still incomplete.
In the present work, we make a contribution to clarify this problem by extensive Monte Carlo simulations of bottle-brush polymers [34, 35] using the "PrunedEnriched Rosenbluth Method" (PERM algorithm) 38] to obtain the relevant information on the conformation of bottle-brush polymers under various conditions, that are needed to test the phenomenological models used to interpret the experimental scattering data [3, 4, 5]. As was shown in 34] the PERM algorithm is very powerful to obtain a wealth of simulation data for the case of side chains grafted to a hard rod (a generalization of the algorithm to a flexible backbone is far from trivial), representing a strictly rigid backbone polymer. This idealization describes a real bottle-brush chain only locally. However, all theoretical models used for the analysis of experiments [3, 4, 5] determining the structure of a bottle-brush do contain the rigid backbone as a special case. It is this case for which we can undertake a stringent test of physical model assumptions underlying the analysis of experimental data. Of course, there is no reason to assume that a model that already fails for the (simpler) rigid backbone case should be accurate for bottle-brushes with flexible backbones.

In Sec. II, we introduce our model and recall the most basic facts on our simulation method and define the quantities that are studied. In Sec. III, we give a comprehensive overview of our results on various physical properties of the bottle-brush polymers. Sec. IV then is devoted to the problem relevant for the interpretation of the experiments, namely the test of theoretical models used in [3, 4, 5, 6] for our system: Note that unlike the experiment, we can extract information of radial density profiles and geometrical characteristics of individual side chains directly from the simulation, simultaneously with but independent of the information gathered on the scattering functions, and thus a stringent test of the proposed 
relations between these quantities is possible. Sec. V then summarizes our conclusions.

\section{MODEL AND SIMULATION METHODOLOGY}

As in Ref. [34], we use as a coarse-grained simple model of flexible polymers in solution, the self-avoiding walk on the simple cubic lattice. Each lattice site can be occupied by a single effective monomeric unit only, and this excluded volume interaction corresponds to polymer chain conformations under good solvent conditions [39, 40, 41]. Introducing an energy parameter $\epsilon$ that is won if two effective monomers occupy nearest neighbor sites, one can describe variable solvent quality in this model simply by varying the temperature $T$ : the Theta temperature $\Theta$ where this attraction approximately cancels the excluded volume repulsion, in the sense that the mean square gyration radius $\left\langle R_{g}^{2}\right\rangle_{T}$ of a chain scales linearly with the chain length $N$, apart from logarithmic corrections [39], occurs for $q_{\Theta}=\exp \left(-\epsilon / k_{B} \Theta\right)=1.3087$ [38]. We shall present results both for $T=\Theta$ and for $T \rightarrow \infty$ where $q=\exp \left(-\epsilon / k_{B} T\right)=1$, and hence only the excluded volume interaction is present $\}$, in view of the fact that most cases of experimental interest will be somewhere in between these limits.

Following Ref. 34] we take the rigid backbone along the $z$-axis of our coordinate system. Using even values for the length $L_{b}$ of the backbone, measuring all lengths in units of the lattice spacings, grafting sites $z=1$, $z=L_{b}$ at the end of the backbone are labeled as $s_{1}$, sites adjacent to the ends $\left(z=2, z=L_{b-1}\right)$ as $s_{2}$, and so on, until the center of the bottle-brush, sites $z=L_{b} / 2$, $z=L_{b} / 2+1$ being denoted as $s_{L_{b} / 2}$. Of course, a dependence of properties of a side chain on the coordinate $s_{k}$ occurs only when we consider bottle-brush polymers with free ends in the $z$-direction, while no dependence on $s_{k}$ occurs if we choose periodic boundary conditions (pbcs) in $z$-direction such that the grafting site $z=L_{b}$ is nearest neighbor of $z=1$ : in this case full translational invariance in $z$-direction holds, and the distribution function of the monomers $P_{z^{\prime}}\left(z-z^{\prime}\right)$ of a side chain grafted at $z^{\prime}$ must be symmetric around $z^{\prime}, P_{z^{\prime}}\left(z-z^{\prime}\right)=P_{z^{\prime}}\left(z^{\prime}-z\right)$. This symmetry property does not hold only for the distribution function of all the monomers that belong to that side chain, but also for individual monomers $i=1, \ldots, N$ along the side chain, in particular for the chain ends. Also the average $z$-coordinate of the center of mass of the side chain coincides with $z^{\prime}$. None of these symmetries carry over to the case with free ends, of course; in the latter case the monomers of the side chain can have $z$-coordinates in the region $-N+1 \leq z \leq L_{b}+N$ (the boundaries of this interval do not occur in practice, of course, it would require that a side chain grafted at $z=1$ or $z=L_{b}$ is linearly stretched out in the $-z$ or $+z$-direction, respectively).

When one considers properties of individual side chains, which are stretched away from the backbone, two non-equivalent directions $x, y$ need to be distinguished [34]: defining the vector toward to the center of mass (C.M.) of a chain from its grafting point $z^{\prime}$ as $\left(X_{z^{\prime}}, Y_{z^{\prime}}, Z_{z^{\prime}}\right)$ in a fixed laboratory frame, for a particular configuration of the side chain, we can define the $x$-axis along the vector $\left(X_{z^{\prime}}, Y_{z^{\prime}}\right)$, and require that the $y$-axis is perpendicular to the $x$-axis and also lies in the $X_{z^{\prime}}-Y_{z^{\prime}}$ plane. Since for a densely grafted bottlebrush polymer strong stretching of the side chains is expected [8, 9, 10, 11, 12, 13, 19] this distinction allows to compute linear dimensions of the side chains in the direction along which the chain is stretched, and perpendicular to it.

In practice, side chain lengths up to $N=50$ were considered, while choices $L_{b}=32,64,128$ and 256 as well as two values of the grafting density, $\sigma=1 / 2$ and $\sigma=1$, were considered. A distinctive feature of our implementation of the PERM algorithm is [34] that in one run one gets information on properties for all integer values of $\mathrm{N}$ from $N=1$ up to $N_{\max }$ \{which in our case was chosen to be $N_{\max }=50$, so the largest polymer simulated had a total number of monomers $\mathcal{N}_{\text {tot }}=L_{b}+L_{b} \sigma N=256+(256 / 2) 50=6656$, since for $L_{b}=256$ the case $\sigma=1$ no longer was feasible $\}$. For details on the implementation of the PERM algorithm for bottle-brush polymers, we refer the reader to Ref. [34].

\section{STRUCTURAL PROPERTIES OF BOTTLE BRUSHES: THE EFFECT OF CHAIN ENDS}

In this section we will look at the difference in structure at the free ends of the backbone, where we can expect to find star-like conformations for the side chains, and the central part of the backbone which will be brushlike. Comparing conformations for bottle-brushes with free ends to those where pbcs are employed along the rigid backbone, we can also find out to what extend the free ends influence the average structure of the brush and its side chains. We will perform this comparison for good solvent as well as for theta-solvent conditions.

Fig. 1 presents our data for the perpendicular part of the mean square gyration radius, $\left\langle R_{g, \perp}^{2}\right\rangle$, where $R_{g, \perp}^{2} \equiv$ $R_{g, x}^{2}+R_{g, y}^{2}$, and the $x$ and $y$-components refer to "measurements" taken in the laboratory system with fixed orientations of the coordinate axes along the axes of the simple cubic lattice. One sees that $\left\langle R_{g, \perp}^{2}\right\rangle$ for the grafting density $\sigma=1$ is always larger than for $\sigma=1 / 2$, while the dependence on backbone length is almost invisible. In the good solvent case, data for the decade $5 \leq N \leq 50$ are compatible with a power law increase, but the exponent is far too small in comparison with the prediction of the scaling theory for large enough $N$ and high grafting density one expects 34] $\left\langle R_{g, \perp}^{2}\right\rangle / N^{2 \nu} \propto N^{2 \nu(1-\nu) /(1+\nu)} \approx$ $N^{0.305}$ while the effective exponents that one can read off from Fig. 1a are only about half of this value\}. Interestingly, also in the $\Theta$-solvent case one observes an 
(a)

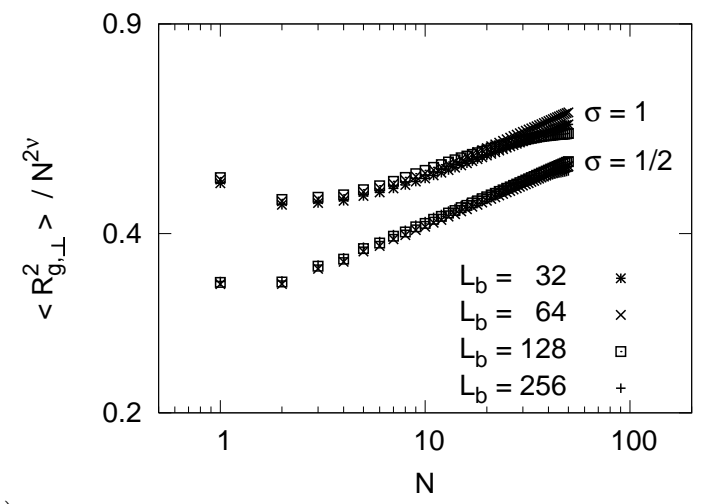

(b)

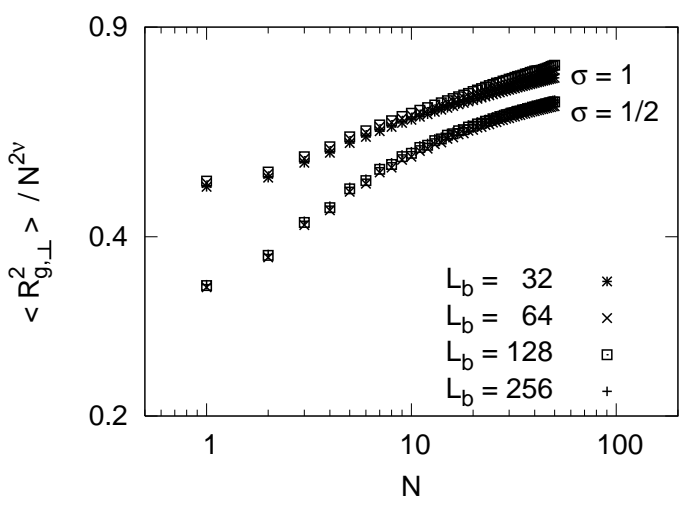

FIG. 1: Log-log plot of the rescaled mean square gyration radius perpendicular to the backbone, $\left\langle R_{g, \perp}^{2}\right\rangle / N^{2 \nu}$ of the whole bottle-brush versus the side chain length, for a good solvent where $\nu=0.588$ (a) and a $\Theta$-solvent where $\nu=0.5$ was taken (b). Two choices of $\sigma$ and four choices of $L_{b}$ are included, as indicated. All data are for bottle-brushes with free ends.

increase of $\left\langle R_{g, \perp}^{2}\right\rangle / N^{2 \nu}=\left\langle R_{g, \perp}^{2}\right\rangle / N$ with increasing side chain length $N$, but there clearly occurs curvature on the log-log plot, and thus already the data indicate that the asymptotic region where power laws and scaling concepts apply is not reached. Analogous data have also been taken for the model with pbcs, but the data are almost indistinguishable from the free end case, and hence not shown here.

Fig. 2 now turns to the linear dimensions of side chains, using a coordinate system where the $x$-direction is defined from the direction of the vector through the backbone and the C.M. of each side chain, and perpendicular to the backbone direction in each configuration (see Sec. II), and also different grafting sites are distinguished, for a relatively short backbone length, $L_{b}=32$. As expected (Fig. 2 2 ), the stretching of chains grafted near the free ends $\left(s_{1}\right)$ in radial direction is weakest, because they acquire a noticeable component in the $z$-direction (Fig. 2r). These effects rather quickly get weaker when the grafting site is farther away from the chain ends, and even for a short backbone $\left(L_{b}=32\right)$ the chains near the center almost behave like chains in the bulk of a very long chain (which is modeled by eliminating end effects through the choice of pbcs). We do not have such an obvious interpretation for the weak (but for the backbone ends clearly non-monotonic) variation of $\left\langle R_{g, y}^{2}\right\rangle / N^{2 \nu}$, however.

It is interesting to contrast these results to the $\Theta$ solvent (Fig. 3). In this case the inhomogeneity caused by the presence of free ends of the backbone is much weaker, the differences with respect to the p.b.c. case are much less significant. However, a rather strong effect of the inhomogeneity in the $z$-direction is seen when one considers the corresponding components of the mean square backbone to end distance of the side chains, and this effect is present both in the excluded volume case and in the $\Theta$-solvent case (Fig. (4).

The next question we ask is the following: how likely is it that monomers (or chain ends) are not in the region $1 \leq z \leq L_{b}$ where the grafting sites are? Fig. 5 shows also from this criterion that in the good solvent case the bottle-brush is more extended in the $z$-direction than in the $\Theta$-solvent case. Even for short backbones $\left(L_{b}=32\right)$ for $\Theta$-solvents bulk behavior is reached, while for good solvents there is still some systematic depression in the center $\left(z=L_{b} / 2=16\right)$. We note, however, that for larger $L_{b}$ such as $L_{b}=64$ (to save space these data are not shown) bulk behavior is reached for a significant range of $z$ in the center of the bottle-brush.

Another quantity that shows that side chains near the backbone ends tend to orient much more along the $z$-axis in the good solvent case rather than in the $\Theta$-solvent case is the distribution $P(\theta)$ of the angle between the vectors towards the center of mass of each side chain and the $z$-direction (Fig. 6). One should note that angles $\theta$ near $\theta=\pi / 2$ characteristic for chains stretched away from the backbone in perpendicular direction, dominate only in the center of the backbone, while angles near $\theta=\pi / 4$ and $3 \pi / 4$ make a substantial contribution near the backbone ends. For the considered side chain length, this effect dies out after a few monomeric distances away from the backbone ends, however. For $\Theta$-conditions (Fig. 6b) this behavior is only found close to the chain end. Chains grafted already five monomers away from the backbone end show no tilting like for the good solvent case. The average angle remains at $\pi / 2$, but the distribution gets broader and asymmetric with a heavy tail towards the adjacent chain end.

The data shown in Figs. 20 are readily accessible in simulation, but not easy to access experimentally. They help, nevertheless, to develop a complete picture of the structure of bottle-brush polymers and clarify the side chain conformations. Quantities, that experimentalists try to extract from their studies are accessible to the simulations as well, of course. Such quantities are the radial distribution $\rho(r)$ of the monomers and $\rho_{e}(r)$ of the chain ends (shown in Figs. 7, 8 for $L_{b}=64$ ). Note that due to the discreteness of the lattice, the number of monomers, $N(r)$ and $N_{e}(r)$, in the interval $[r, r+d r]$ are normalized i.e. $\rho(r)=N(r) / N_{r}$ and $\rho_{c}(r)=N_{e}(r) / N_{r}$ where 
(a)

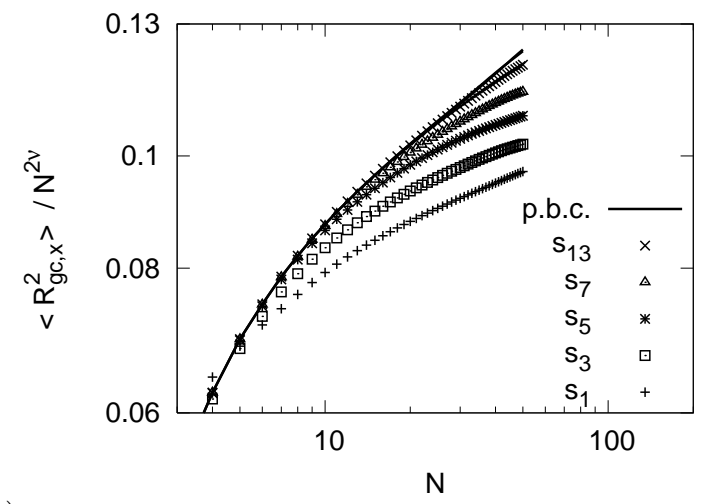

(b)

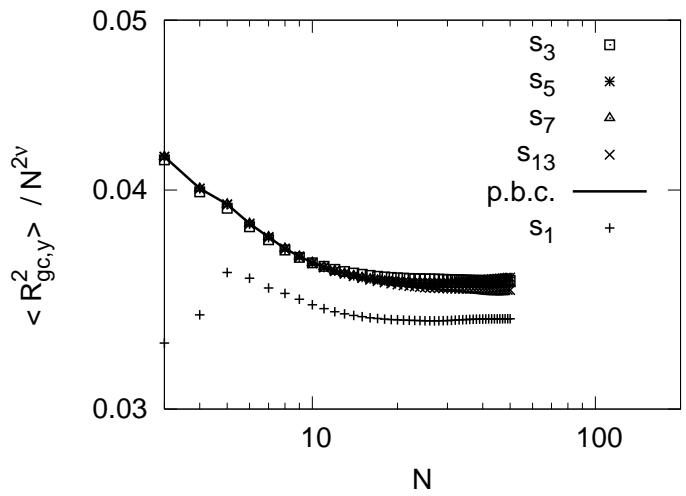

(c)

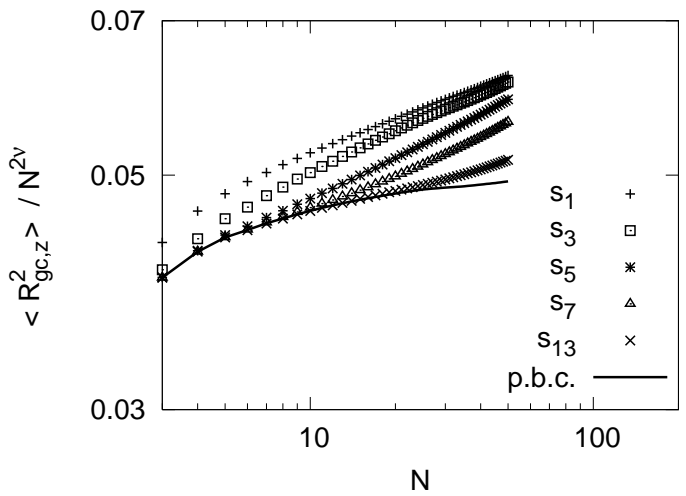

FIG. 2: Log-log plot of the rescaled mean square gyration radii of the side chains, $\left\langle R_{g c, x}^{2}\right\rangle / N^{2 \nu}(\mathrm{a}),\left\langle R_{g c, y}^{2}\right\rangle / N^{2 \nu}(\mathrm{b})$ and $\left\langle R_{g c, z}^{2}\right\rangle / N^{2 \nu}$ (c) versus the side chain length $\mathrm{N}$, for the good solvent case, $L_{b}=32, \sigma=1$ and various choices of the grafting sites, as shown by the coordinate $s_{k}$ (cf. Sec. II for explanations). The full curves show analogous data for the case of pbcs.

$N_{r}$ is the number of lattice points with a distance to the backbone lying in the interval $[r, r+d r]$. For comparing data of different chain lengths, normalization conditions $\sum_{r} N(r)=N$ and $\sum_{r} N_{e}(r)=n_{c}$ have been imposed. Similar data have also been generated for $L_{b}=32$, but the differences to those shown are only small, and therefore need not be discussed here. Figs. 7 and 8 reveal that neither $\rho(r)$ nor $\rho_{e}(r)$ are sensitive to the effects of the free ends: for the chosen $L_{b}$, much longer side chains would be required in order that effects due to the crossover from bottle-brush to star polymer behav- ior come into play. While in the good solvent case the chain ends are typically farther away from the backbone than in the $\Theta$-solvent case, the qualitative behavior of $\rho(r)$ and $\rho_{e}(r)$ does not depend on solvent quality much. Furthermore, it is gratifying to note that these data are qualitatively rather similar to the corresponding Molecular Dynamics results of Murat and Grest [14] for a beadspring off lattice model of flexible side chains tethered to a straight line. This similarity reinforces our view that on a coarse grained level, the present lattice model should yield useful results. 
(a)

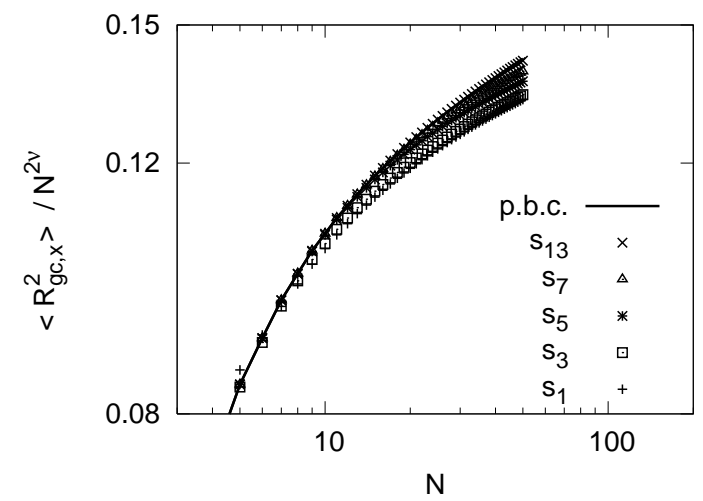

(b)

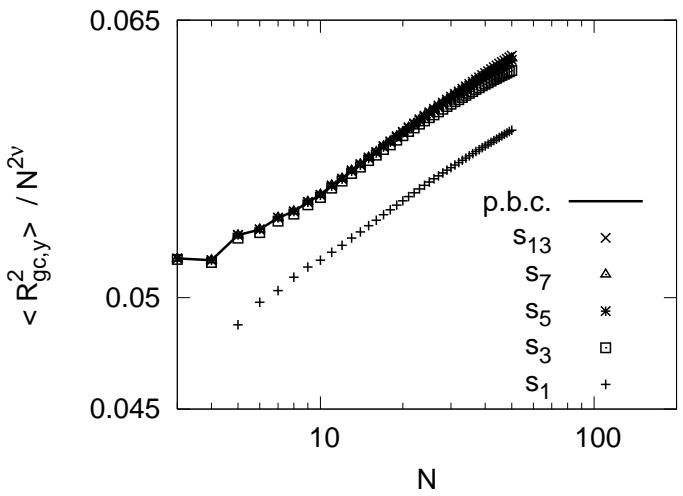

(c)

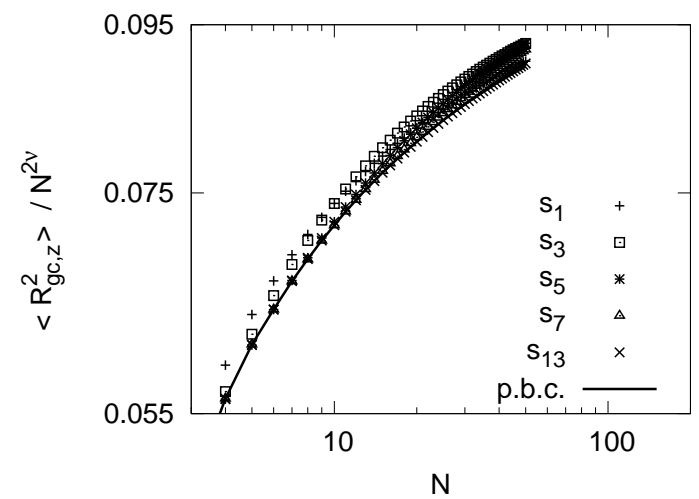

FIG. 3: Same as Fig. 2, but for the case of the $\Theta$-solvent.

\section{SCATTERING FUNCTIONS FOR BOTTLE-BRUSH POLYMERS AND THEIR THEORETICAL MODELING}

Let us now turn to a discussion of experimentally observable information on the structure of a bottle brush polymer. In experiments one has to infer the structure from scattering data [3, 4, 5] employing suitable model assumptions on the structure. In the simulation we obtain both, the scattering data and the underlying structural properties described in the last section independently, and therefore are able to test theoretical models suggested to link the two. Fig. 9 presents our data for the total scattering function $S_{\mathrm{w}}(q)$ for the bottle-brush polymers, both for good solvent and $\Theta$-solvent conditions. Here $S_{\mathrm{w}}(q)$ is defined as

$$
S_{\mathrm{W}}(q)=\frac{1}{\mathcal{N}_{\text {tot }}} \sum_{i=1}^{\mathcal{N}_{\text {tot }}} \sum_{j=1}^{\mathcal{N}_{\text {tot }}}\left\langle c\left(\vec{r}_{i}\right) c\left(\vec{r}_{j}\right)\right\rangle \frac{\sin \left(q\left|\vec{r}_{i}-\vec{r}_{j}\right|\right)}{q\left|\vec{r}_{i}-\vec{r}_{j}\right|},
$$

where $c\left(\vec{r}_{i}\right)$ is an occupation variable, $c\left(\vec{r}_{i}\right)=1$ if the site $\vec{r}_{i}$ is occupied by a bead, and zero otherwise. Note that an angular average over the direction of the scattering vector $\vec{q}$ has been performed, and the sums run over all monomers (all side chains and the backbone).

Surprisingly, our data are qualitatively very similar 
(a)

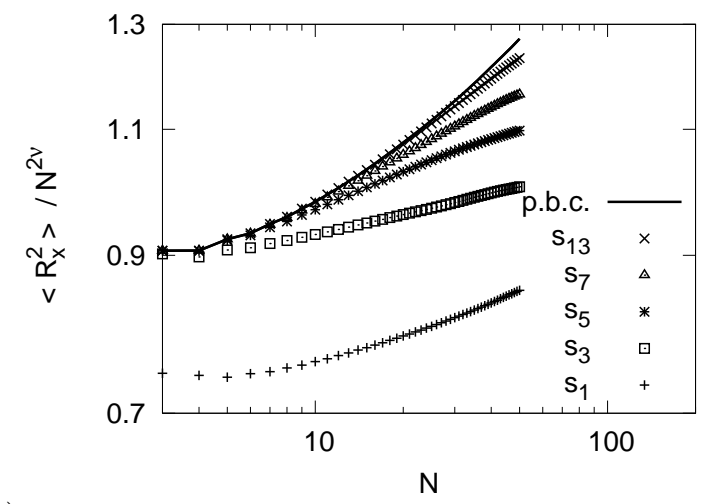

(c)

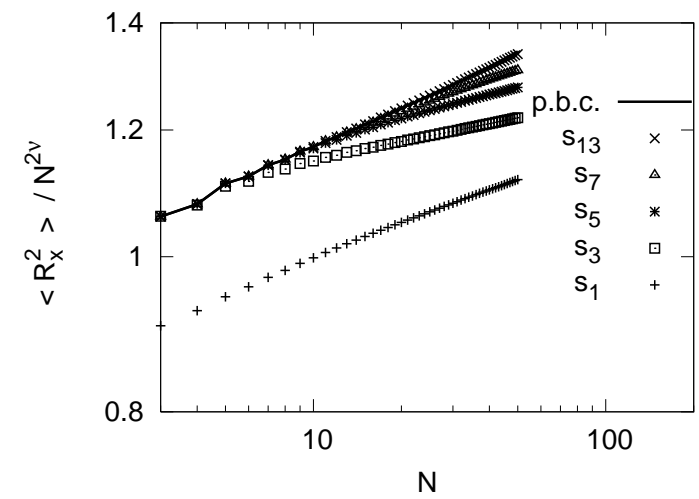

(b)

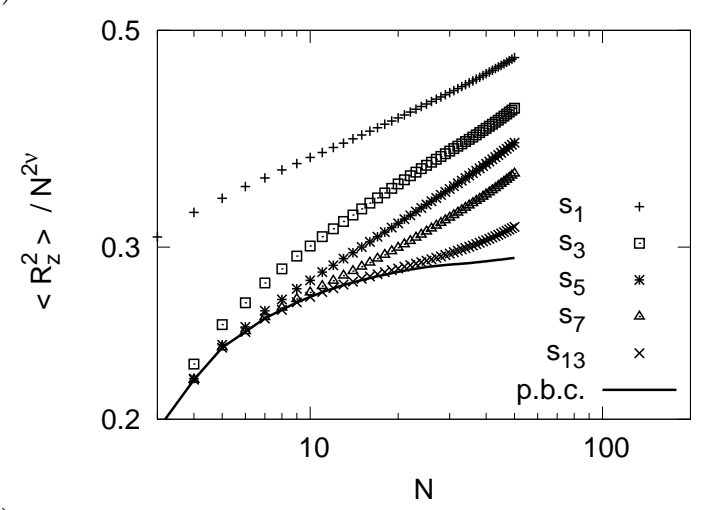

(d)

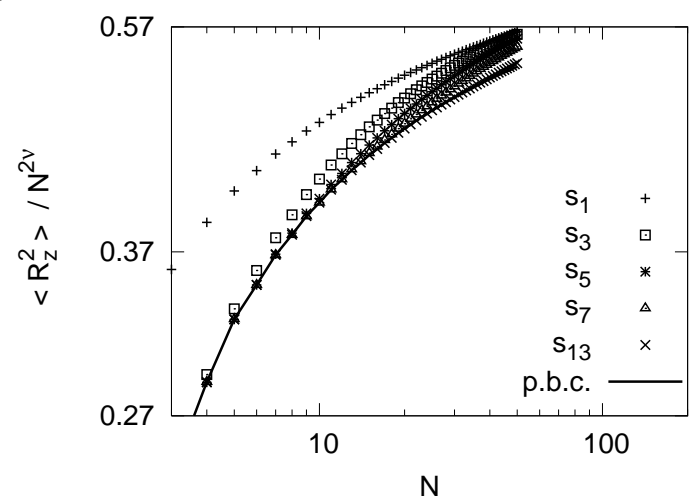

FIG. 4: Log-log plot of the mean square backbone-to-end distance of the side chains versus the side chain length $N$, for $L_{b}=32$ and $\sigma=1$. Panels (a,b) refer to the good solvent, panels (c,d) to the $\Theta$-solvent case. The components shown are $\left\langle R_{x}^{2}\right\rangle / N^{2 \nu}$ $(\mathrm{a}, \mathrm{c})$ and $\left\langle R_{z}^{2}\right\rangle / N^{2 \nu}(\mathrm{b}, \mathrm{d})$. Symbols denote different coordinates $s_{k}$ along the backbone, while the full curves show the analogous result for pbcs.

to the corresponding experimental data (see e. g. Fig. 4 of [3]), although the latter refer to a polymer with a flexible backbone, unlike our simulations. As always, the limit $q \rightarrow 0$ of $S_{\mathrm{w}}(q)$ reflects the total number $\mathcal{N}_{\text {tot }}$ of scattering monomers, and the leading deviation from it is described by the total gyration radius,

$$
S_{\mathrm{w}}(q) \approx \mathcal{N}_{\text {tot }}\left[1-q^{2}\left\langle R_{g}^{2}\right\rangle / 3\right] .
$$

This behavior is shown by the fine-dotted lines in Fig. 9 for the case of grafting density $\sigma=1$. The $q$ range over which this approximation agrees with the scattering data increases with increasing ratio of side chain length to backbone length, $N / L_{b}$. Of course, more interesting is the behavior at larger $q$, where Eq. (2) is no longer valid. The region where $S_{\mathrm{w}}(q)$ is strongly curved and decreases rapidly ( $0.1 \leq q \leq 0.3$, in our case) has contributions from the conformation along the backbone (rigid rod in our case which should show up as a behavior $S \mathrm{w}(q) \simeq q^{-1}$ for longer backbones) and from the scattering from the cross section through the cylindrical bottle-brush, and needs to be related to data such as shown in Figs. 7.8
The $q$ range near $q=1$ reflects the self-avoiding walk structure $q^{-1 / \nu}$ before it is affected by the local packing of monomers on the lattice at still larger $q$, and in real systems reflects local properties such as the persistence length of the flexible side chains, possible scattering from side groups, etc. This non-universal regime hence is less interesting. From this discussion of the total structure factor we can already conclude that it is the $q$-range $0.04 \leq q \leq 0.5$ which for our model contains the important information about the structure of the brush.

One advantage of our simulations is that we can obtain scattering contributions from different parts separately. E. g., we can isolate the scattering from the backbone (Fig. 10 a ) and from the scattering of the side chains (Fig. 10b,c). In our case, where the backbone is a rigid rod where just the subsequent sites $i=1,2, \ldots, L_{b}$ are taken by monomers, Eq. (11) becomes

$$
S_{\mathrm{b}}(q)=\frac{1}{L_{b}} \sum_{i=1}^{L_{b}} \sum_{j=1}^{L_{b}} \frac{\sin (q|j-i|)}{q|j-i|} .
$$


(a)

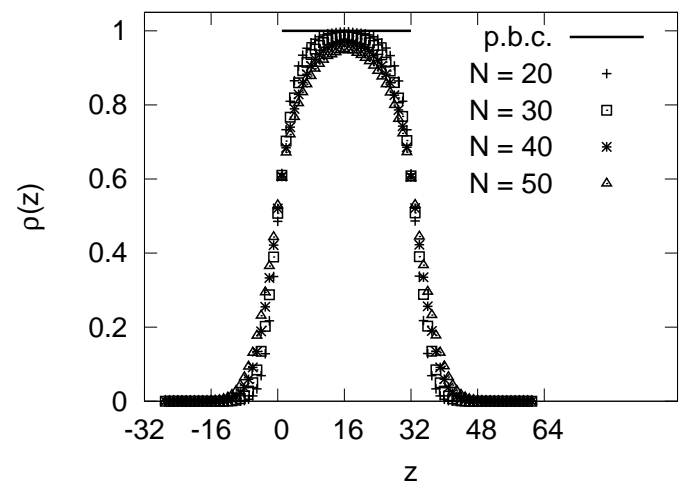

(c)

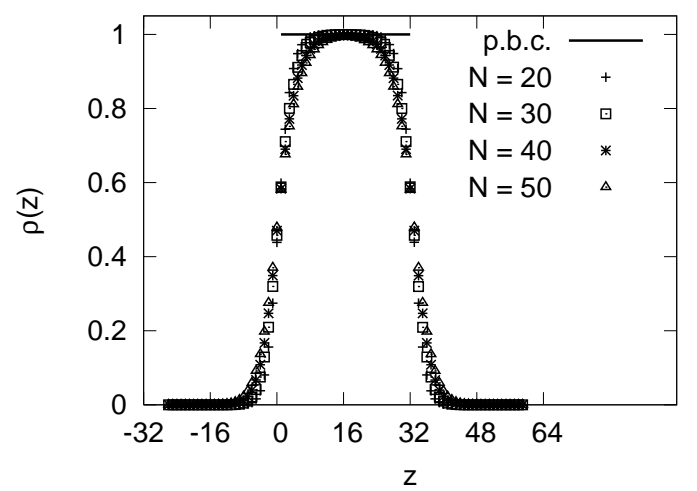

(b)

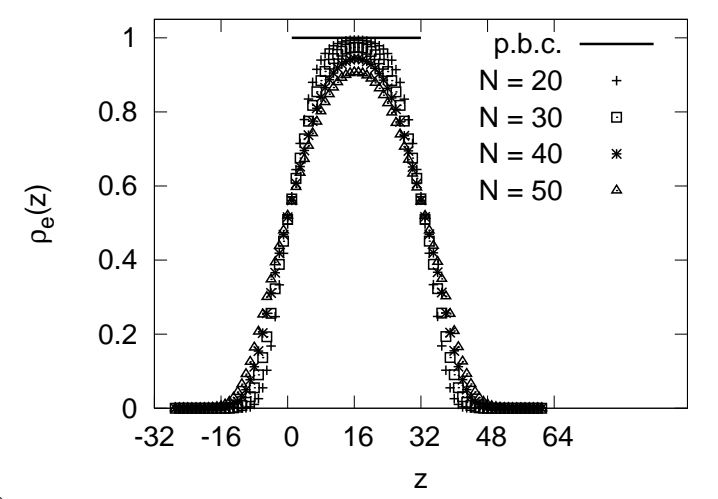

(d)

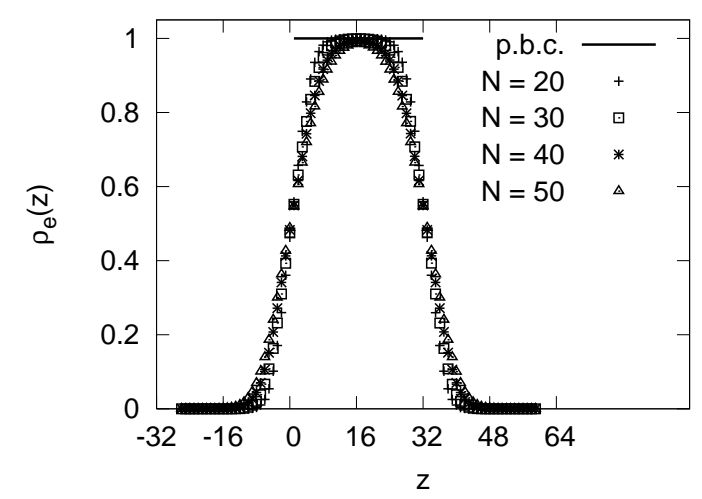

FIG. 5: Density distributions of all the monomers, $\rho(z)$, (a,c), and of the chain ends, $\rho_{e}(z)$ (b,d), plotted vs. $z$ for $L_{b}=32$, $\sigma=1$, good solvent conditions (a,b) and $\Theta$-solvent conditions (c,d). The distributions are normalized by choosing $\sum_{z} \rho(z)=n_{c}$ and $\sum_{z} \rho_{e}(z)=n_{c}$, where $n_{c}$ is the number of side chains $\left(n_{c}=\sigma L_{b}\right)$. Four chain lengths are shown, as indicated. Note that in the p.b.c. case we trivially have $\rho(z)=\rho_{e}(z)=1,1 \leq z \leq L_{b}$, for the chosen normalization.

Noting that the distance $|j-i|=0$ occurs $L_{b}$ times, while the distance $|j-i|=1$ occurs $2\left(L_{b}-1\right)$ times, the distance $|j-i|=2$ occurs $2\left(L_{b}-2\right)$ times, etc., we conclude that

$$
S_{\mathrm{b}}(q)=-1+\frac{2}{L_{b}} \sum_{k=0}^{L_{b}-1}\left(L_{b}-k\right) \frac{\sin (q k)}{q k} .
$$

The factor 2 accounts for the fact that both positive and negative differences $k=j-i$ occur, and the extra -1 corrects for over counting in the term $k=0$.

In the limit where $L_{b} \rightarrow \infty$ and $q L_{b}$ is of order unity, the sum in Eq. (4) can be transformed into an integral, to find

$$
S_{\mathrm{b}}(q) \approx \frac{2}{q} \int_{0}^{q L_{b}} \frac{\sin t}{t} d t-4 \frac{\sin ^{2}\left(q L_{b} / 2\right)}{q^{2} L_{b}}
$$

Eq. (5) is nothing but the well-known scattering function of an infinitely thin rod of length $L_{b}$ with a continuous mass distribution along the rod [42, 43].
According to Eq. (11), the scattering function of all side chains is given by

$$
S_{\mathrm{s}}(q)=\frac{1}{N n_{c}} \sum_{i=1}^{N n_{c}} \sum_{j=1}^{N n_{c}}\left\langle c\left(\vec{r}_{i}\right) c\left(\vec{r}_{j}\right)\right\rangle \frac{\sin \left(q\left|\vec{r}_{i}-\vec{r}_{j}\right|\right)}{q\left|\vec{r}_{i}-\vec{r}_{j}\right|} .
$$

When we add $S_{\mathrm{b}}(q)$ and $S_{\mathrm{s}}(q)$ with their relative weights, see Figs. 10a,b, we do not recover $S_{\mathrm{w}}(q)$ strictly, due to interference effects in the scattering from monomers in the side chain and in the backbone. Such interference effects normally are neglected $[\underline{3}, 4,[4,[5]$. Taking the difference $S_{b s}=\left[N_{\text {tot }} S_{\mathrm{w}}(q)-L_{b} S_{b}(q)-N n_{c} S_{s}(q)\right] /\left(2 N_{\text {tot }}\right)$ , we can test for the importance of such interference effects as shown in Fig. 11. Indeed, we do find that such interference effects are present although only at a percent level.

We now turn to a discussion of the scattering from the side chains, which clearly dominates the scattering intensity in all cases of practical interest. For scattering wavenumber $q$ in the range $q\left\langle R_{g}^{2}\right\rangle \gg 1$ this scattering should be dominated by the cross sectional structure 
(a)

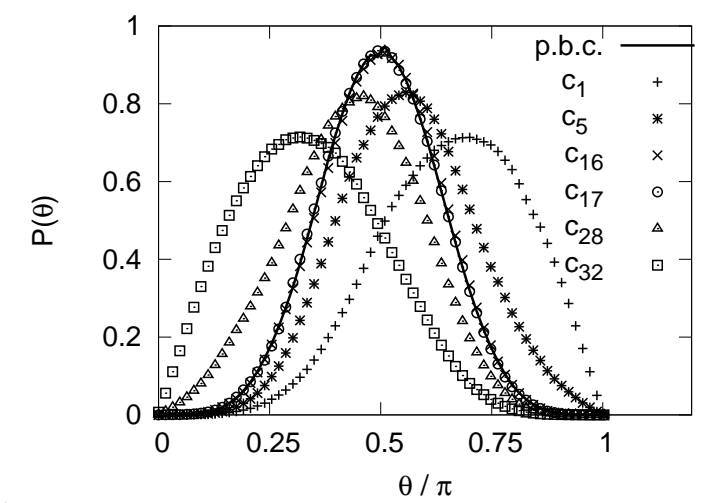

(b)

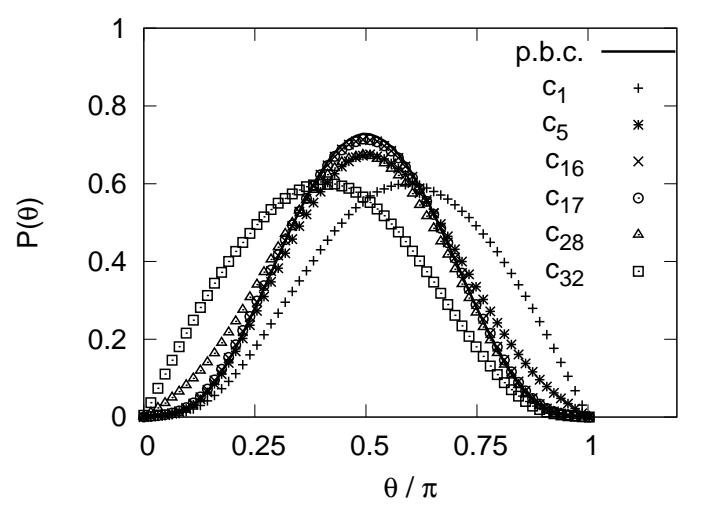

FIG. 6: Distribution $P(\theta)$ of the angle $\theta$ between the vectors towards the center of mass of each side chain and the direction of the backbone, for $L_{b}=32, \sigma=1, N=50$, the good solvent case (a) and the $\Theta$-solvent case (b). The different symbols indicate different positions along the backbone, as indicated. The corresponding distribution for the p.b.c. case agrees with the $c_{16} / c_{17}$ curves.

of the bottle-brush. In the analysis of the experimental scattering data one has to assume that one can determine the cross-sectional contribution by a factorization

$$
S_{\mathrm{w}}(q) \equiv S_{\mathrm{b}}(q) S_{\mathrm{xs}}(q),
$$

where $S_{\mathrm{xs}}(q)$ is interpreted as the cross section structure factor. Such decoupling approximations seem to be successful for worm-like micelles [42]. In the literature, $S_{\mathrm{b}}(q)$ is modeled by a superposition of rigid rod and worm-like chain form factors, needed to account for backbone bending [6]. In our case we can take Eq. (7) simply as a definition of $S_{\mathrm{xs}}(q)$ using $S_{\mathrm{b}}(q)$ which is known exactly for our case (see Eq. (44)).

The cross sectional scattering is then assumed to be obtainable from a rotationally averaged two-dimensional Fourier transform of the radial density distribution.

$$
S_{\mathrm{XS}}(q)=\frac{1}{C}\left\langle\left|\int d^{2} \vec{r} \rho(\vec{r}) \mathrm{e}^{i \vec{q} \cdot \vec{r}}\right|^{2}\right\rangle_{\mathrm{T}, \hat{\mathrm{q}}} \cdot
$$

(a)

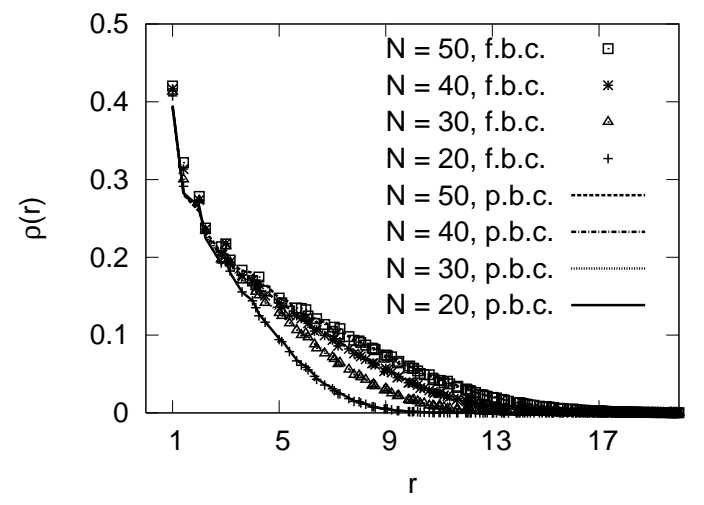

(b)

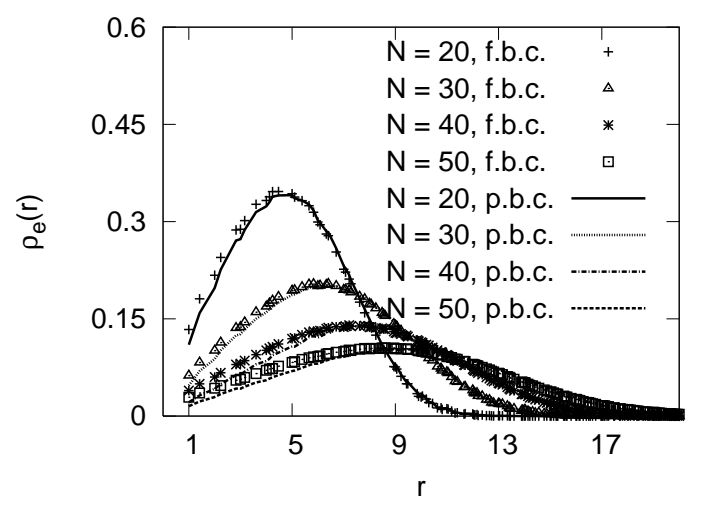

FIG. 7: Radial distribution function $\rho(r)$ of all monomers (a) and radial distribution function $\rho_{e}(r)$ of chain ends (b), plotted versus $r$ for $L_{b}=64, \sigma=1$, good solvent conditions, and four values of the side chain length $\mathrm{N}$, as indicated. Symbols show our results for free ends, while curves show corresponding data for the case of pbcs.

Here $C$ is a normalization, and the indices $T$ and $\hat{q}$ indicate a thermal average and an average over the unit circle in two dimensions. This is further approximated by neglecting correlations in the radial density fluctuations

$$
\left\langle\rho(\vec{r}) \rho\left(\vec{r}^{\prime}\right)\right\rangle_{\mathrm{T}}=\langle\rho(\vec{r})\rangle_{\mathrm{T}}\left\langle\rho\left(\vec{r}^{\prime}\right)\right\rangle_{\mathrm{T}}=: \rho(r) \rho\left(r^{\prime}\right)
$$

to obtain

$$
S_{\mathrm{xS}}(q)=\frac{1}{C}\left|\int d^{2} \vec{r} \rho(r)\left\langle\mathrm{e}^{i \vec{q} \cdot \vec{r}}\right\rangle_{\hat{\mathrm{q}}}\right|^{2}
$$

With the proper normalization this yields

$$
S_{\mathrm{xs}}(q)=\frac{\left|\int_{0}^{\infty} d r r \rho(r) J_{0}(q r)\right|^{2}}{\left|\int_{0}^{\infty} d r r \rho(r)\right|^{2}},
$$

where $J_{0}(r)$ is the zeroth order Bessel function of the first kind. With the approximations underlying Eq.(11) 
(a)

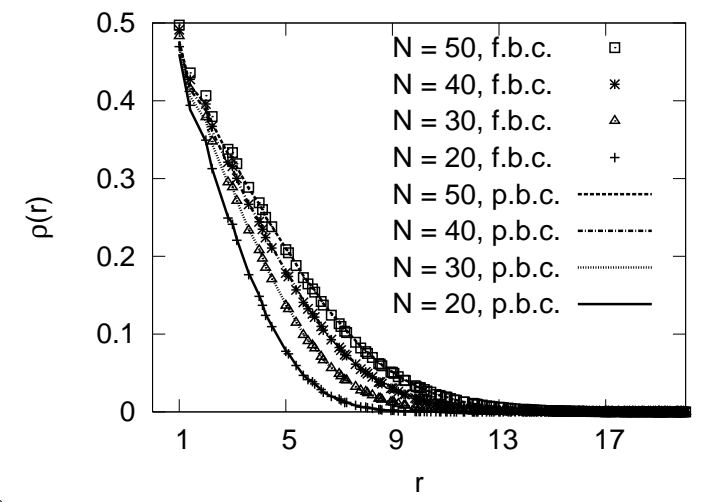

(b)

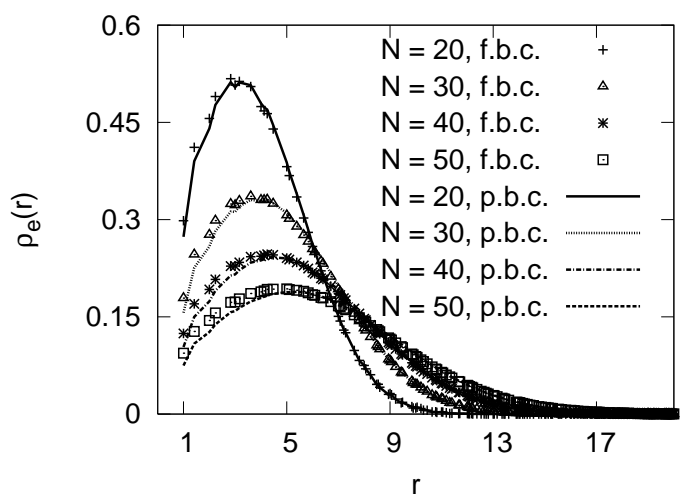

FIG. 8: Same as Fig,. 7 but for $\Theta$-solvent conditions.

the experimental cross section structure factor can be inverted to obtain the radial density distribution

$$
\rho_{\mathrm{xs}}(r)=\frac{1}{2 \pi} \int_{0}^{\infty}\left[S_{\mathrm{xs}}(q)\right]^{1 / 2} J_{0}(q r) q d q .
$$

In the analysis of experimental data, different plausible assumptions for the radial density profile were used, guided by the assumed similarity to the scattering from worm-like micelles. Rathgeber et al. [3] propose to use the following empirical function

$g(r)= \begin{cases}1 & \text { for } r \leq R_{c} \\ \alpha r^{-k}\left\{1+\exp \left[\left(r-R_{s}\right) / \sigma_{s}\right]\right\}^{-1} & \text { for } r>R_{c}\end{cases}$

Here $R_{c}$ is an inner radius, up to which $\rho(r)$ is a constant; then there is a power law decay, described by an exponent $k$, up to some outer radius $R_{s}$, then a fast decay to zero (over the range $\sigma_{s}$ ) follows. The constant $\alpha$ is fixed by the condition that $g\left(r=R_{c}\right)$ is continuous, so Eq. (13) involves the four nontrivial fitting parameters $R_{c}, k, R_{s}$ and $\sigma_{s}$. Zhang et al. [5] assume a form for the cross section structure factor in terms of the first order Bessel function $J_{1}(x)$, (a)

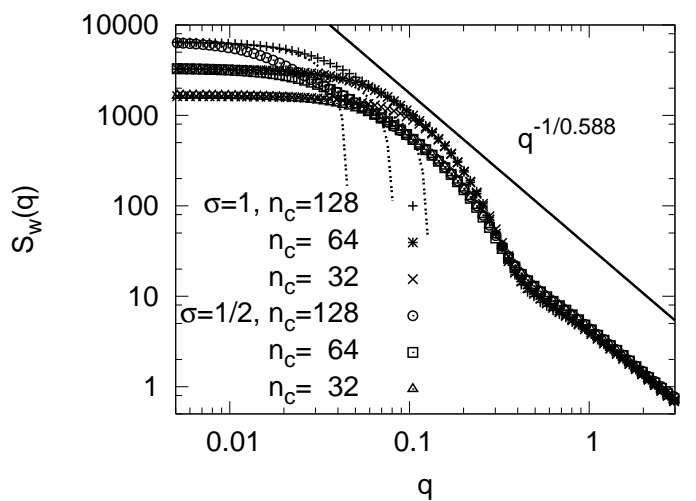

(b)

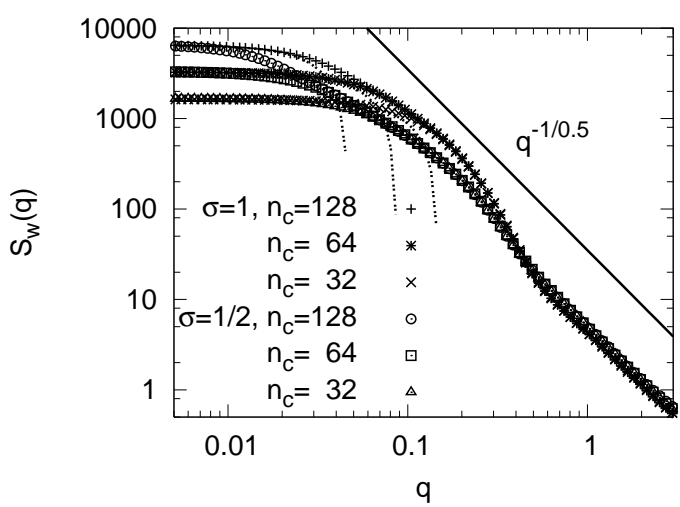

FIG. 9: Log-log plot of the scattering function of the whole bottle-brush polymer, $S_{\mathrm{w}}(q)$, in a good solvent (a) and a $\Theta$ solvent (b) versus $q$. All data are for the case of free ends, $N=50$, while data for two choices of $\sigma$ and three choices of $n_{c}$ each are included, as indicated. Straight lines show the theoretical power laws for flexible chains and intermediate $q$ values, $S_{\mathrm{w}}(q) \propto q^{-1 / \nu}$ and (a) $\nu=0.588$ or (b) $\nu=0.5$ (b), respectively. Dotted curves are given by Eq. (2) for $\sigma=1$.

$$
S_{\mathrm{xs}}(q)=\operatorname{const}\left[\frac{2 J_{1}\left(q R_{c}\right)}{q R_{c}} \exp \left(-q^{2} s^{2} / 2\right)\right]^{2},
$$

where $R_{c}$ is an effective radius, and $s$ is an effective width. This is equivalent to assuming a radial density profile which is a convolution of a step function and a Gaussian

$\rho_{\mathrm{xs}}(r)=\rho_{0} \int d^{2} \vec{r}^{\prime}\left[1-\theta\left(\left|\vec{r}^{\prime}\right|-R_{c}\right)\right] \exp \left[-\frac{\left(\vec{r}-\vec{r}^{\prime}\right)^{2}}{2 s^{2}}\right]$.

Here we have three free parameters, $\rho_{0}, R_{c}$ and $\sigma$. Again, $R_{c}$ is a measure of the range over which the density profile is assumed to be flat in the core of the bottle-brush

When we look at the density profiles determined directly from simulations (see Fig. 12), however, we recognize that there is no convex region in the interior of the bottle brush, even at a grafting density of one which is the limit of what is typically reached in experiment. Therefore, the comparison with worm-like micelles is mislead- 
(a)

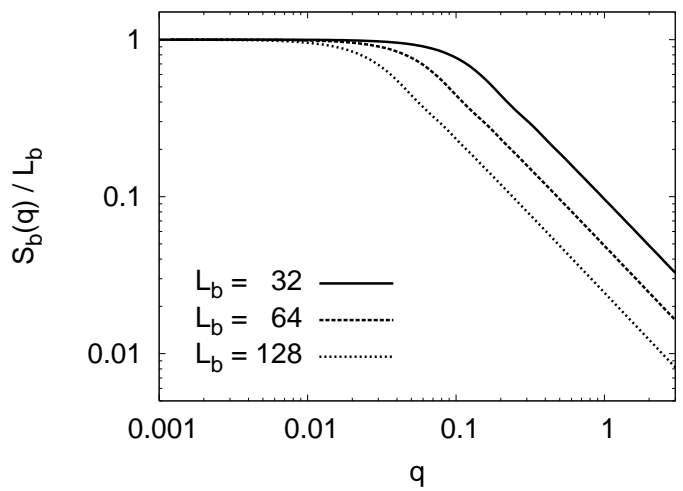

(b)

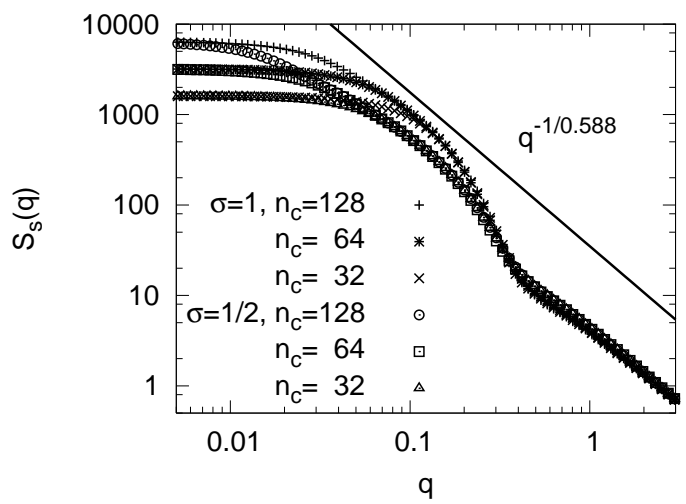

(c)

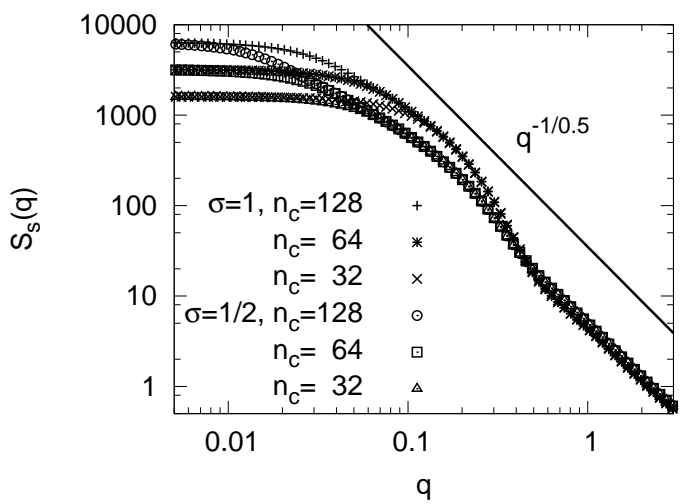

FIG. 10: (a) Log-log plot of the normalized scattering function of the backbone, $S_{\mathrm{b}}(q) / L_{b}$ versus $q$, using the formula of Pedersen and Schurtenberger [42], see Eq. (5) for the case of thin rods. (b) Log-log plot of the scattering from all monomers in the side chains of the bottle-brush with $N=50$. The data are for good solvent conditions, and for two choices of $\sigma$ and three choices of $n_{c}$ each, as indicated. Straight lines have the same meaning as in Fig. 9] (c) Same as (b), but for $\Theta$-solvent conditions. Note that $S_{s}(q)$ is normalized such that $S_{s}(q=0)=N n_{c}$.

ing, and we suggest to use an alternative form of fitting function for the radial density

$$
h(r)=\frac{\sigma}{1+\left(r / r_{1}\right)^{x_{1}}} \exp \left[-\left(r / r_{2}\right)^{x_{2}}\right],
$$

where $\sigma$ is the grafting density and $r_{1}$ and $r_{2}$ are the length scales for the algebraic decay close to the backbone and the exponential cutoff at larger distances (i.e., we expect $r_{1}<<r_{2}$ in the course of our fit analysis), and $x_{1}$ and $x_{2}$ are the corresponding exponents. Taking into account the predictions of scaling theory [34] we can fix the first exponent $x_{1}=(3 \nu-1) / 2 \nu$. So again we are using three fit parameters. In Fig. 12 we show that this assumed form for the radial density is able to fit the simulation data perfectly over almost six orders of magnitude in density for both, good solvent and $\Theta$-solvent conditions. The parameters of the shown fits are $r_{1}=0.49$, $x_{1}=0.65(\nu=0.588), r_{2}=10.67$, and $x_{2}=2.80$ for the good solvent case, and $r_{1}=1.19, x_{1}=0.5(\nu=0.5)$, $r_{2}=7.13$, and $x_{2}=2.18$ for the $\Theta$-solvent case. Within 
(a)

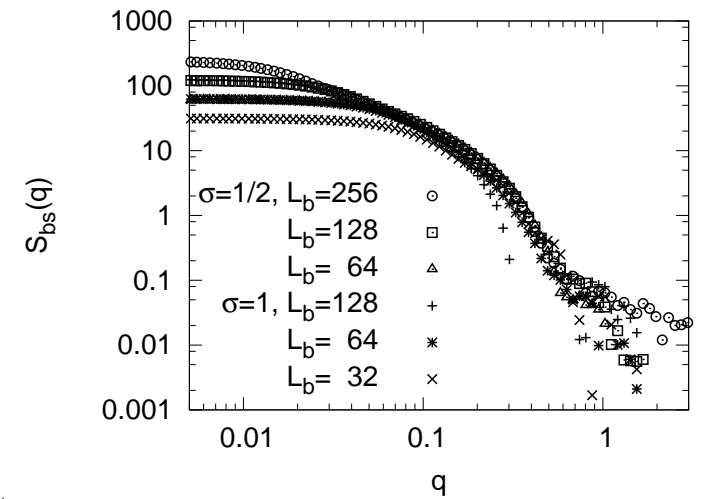

(b)

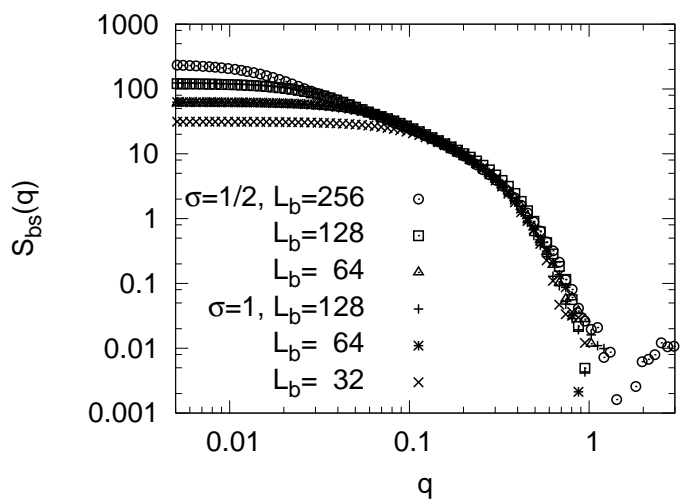

FIG. 11: $S_{\mathrm{bs}}(q)=\left[N_{\mathrm{tot}} S_{\mathrm{w}}(q)-L_{b} S_{b}(q)-N n_{c} S_{s}(q)\right] /\left(2 N_{\text {tot }}\right)$, case (a) is for good solvent conditions, case (b) for $\Theta$-solvent conditions.

the range of backbone lengths studied, the radial density profiles agree, with some statistical fluctuations visible for the good solvent data and the longest backbone, $L_{b}=128$.

Let us now turn to a discussion of the possibility to deduce the radial density profile from the cross sectional structure factor as defined in Eq. (7). Figs. 13a and 14a show fits to the radial density profile using the functional forms $g(r)$ and $h(r)$ defined above. The form $g(r)$ suggested by Rathgeber el al. [3] is able to fit the radial density well over about 3 orders of magnitude with parameters $R_{c}=0.3, k=0.65, R_{s}=10.5$ and $\sigma_{s}=1.90$; $h(r)$ fits over the complete range, as discussed above. When we Fourier transform these functions according to Eq. (11) and compare with the cross sectional structure factor (full lines in Fig. 13b and 14b), we see that the transform only describes the scattering data well up to a momentum transfer value of about $q=0.08$. This is only slightly larger than the range over which one only sees the scattering from the large-scale structure of the bottle brush (Eq. (2)), which fits the cross sectional structure factor up to a momentum transfer value of $q \approx 0.04$, as shown by the dotted line in Fig. 14b. This regime then is basically determined by the normalization of the radial density distribution. Using an iterative optimization (a)

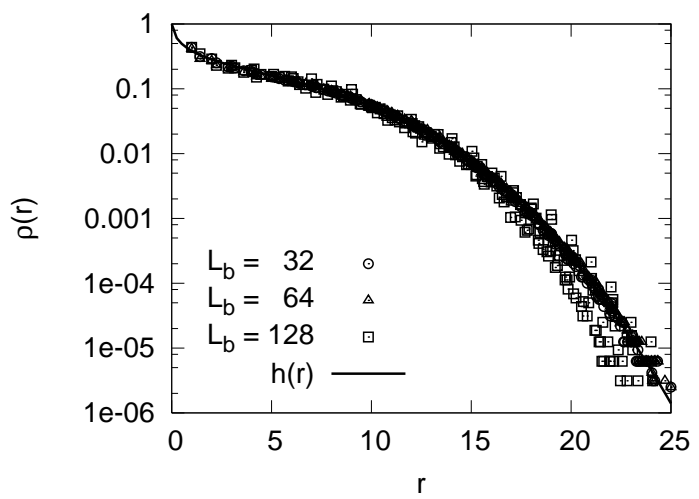

(b)

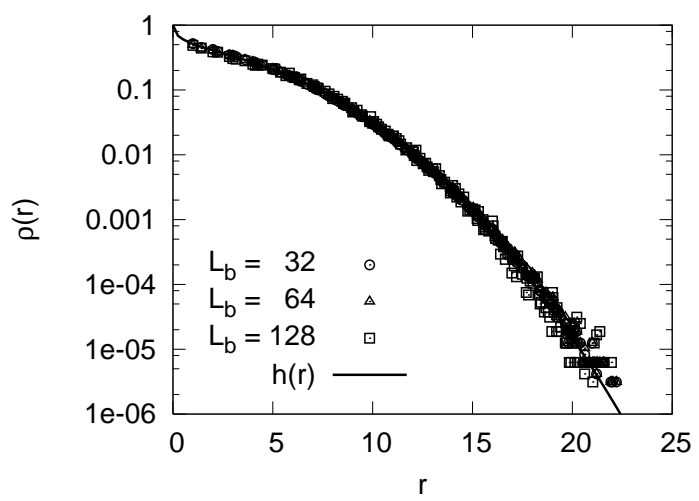

FIG. 12: (a) Radial distribution function $\rho(r)$ plotted versus $r$ for side chain length $N=50$, three choices of backbone length $L_{b}$ as indicated, and the grafting density $\sigma=1$ for good solvent conditions. (b) Same as (a) but for $\Theta$-solvent conditions. Parameters of the fit function $h(r)$ are quoted in the text.

procedure [44] we can also find the best fit of the Fourier transform of the radial densities to the cross sectional scattering shown by the curves indicated as $g_{s}(r)$ (fit parameters are $R_{c}=0.3, k=0.65, R_{s}=7.5$, and $\sigma_{s}=2.8$ ) and $h_{s}(r)$ (fit parameters are $r_{1}=0.49, x_{1}=0.65$, $r_{2}=8.20$, and $\left.x_{2}=1.80\right)$ in Figs. 13b and $14 \mathrm{~b}$, where we extended the fit up to $q \approx 0.4$. When we then look at these functions in real space in Figs 13 and 14a, we see that they are a rather poor fit to the radial density profile. The function $h_{m}(r)$ (fit parameters are $r_{1}=0.49$, $x_{1}=0.65, r_{2}=10.20$, and $\left.x_{2}=2.80\right)$ in Fig. 14 will be discussed later in the text.

Using the functional form of Eq. (14) we can directly fit the data in q-space and then transform into real space. This is shown in Fig. 15 for the good solvent case and in Fig. 16 for the $\Theta$-solvent case. Looking at Figs. 15] and 16] one first has to comment on the fact that the full curves in both figures do not agree with the data given by the symbols. For these curves, the scattering data in the q-range $[0,2 \pi]$ were Bessel transformed into real space and back again. The overestimation of the real scattering for momentum transfers larger than about 0.1 
(a)

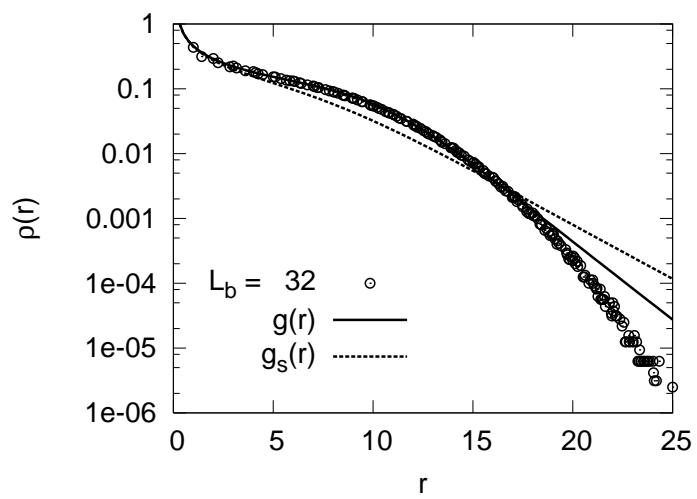

(b)

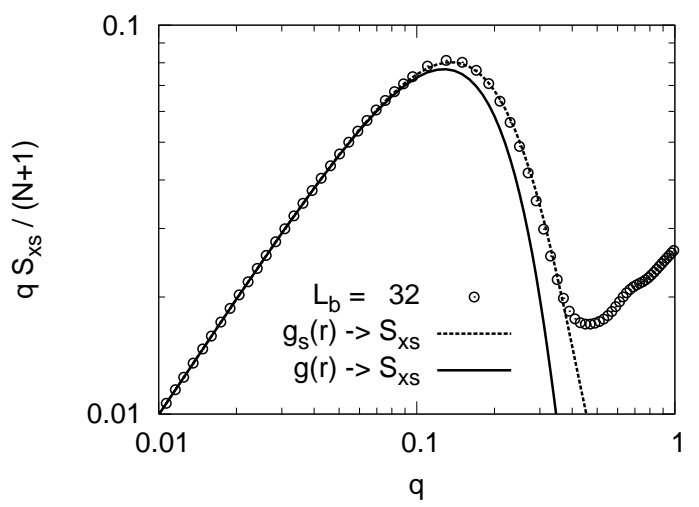

FIG. 13: (a) Radial distribution function $\rho(r)$ plotted versus $r$ for side chain length $N=50$ for good solvent conditions. Parameters of the fit functions $g(r)$ (best fit to $\rho(r)$ ) and $g_{s}(r)$ (Fourier transform of best fit to $S_{\mathrm{xs}}(q)$ ) are quoted in the text. (b) The corresponding cross section structure factor $S_{\mathrm{xs}}(q)=S_{\mathrm{w}}(q) / S_{\mathrm{b}}(q)$ plotted in the representation $q S_{\mathrm{xs}}(q)$, vs. $q$. The two curves correspond to the two curves in part (a).

indicates that there is intensity in the modes for q-values larger than $2 \pi$ which is aliased into the studied range. However, looking at the direct transform of the scattering data into real space $\left(\rho_{\mathrm{xs}}^{(1)}\right.$ in Figs. 15(a) and 16(a)) one sees that this is not a relevant numerical problem. Assuming the whole displayed q-range to be relevant for the determination of the radial density profile leads to the prediction of a highly oscillatory non-positive radial density. Similarly, when we try to fit the scattering data beyond a q-value of about 0.4 by the assumed functional forms $g(r)$ and $h(r)$ we obtain unphysical radial density profiles. Constraining the fit with the functional form of Eq. (14) to the q-range below 0.4, however, also does not lead to a satisfactorily prediction of the radial density as can be seen in Figs. 15a and 16a. The assumed convex shape of the radial density leads to an overestimation of the density in the interior of the brush and a compensating underestimation in the outer parts.

Summarizing this discussion we have to conclude that there is only a small range of momentum transfers where (a)

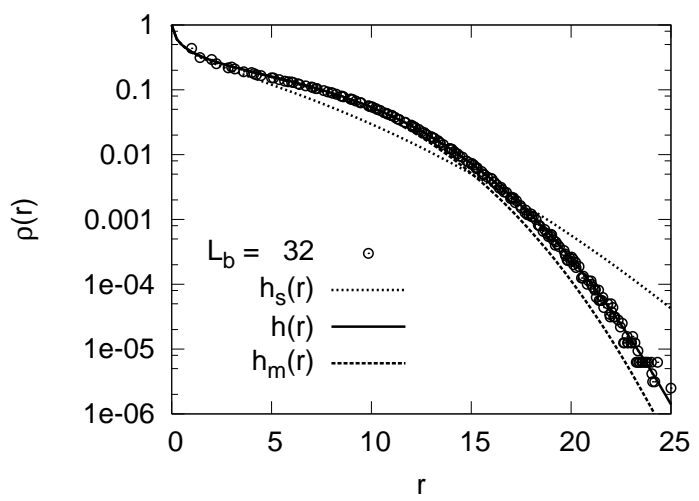

(b)

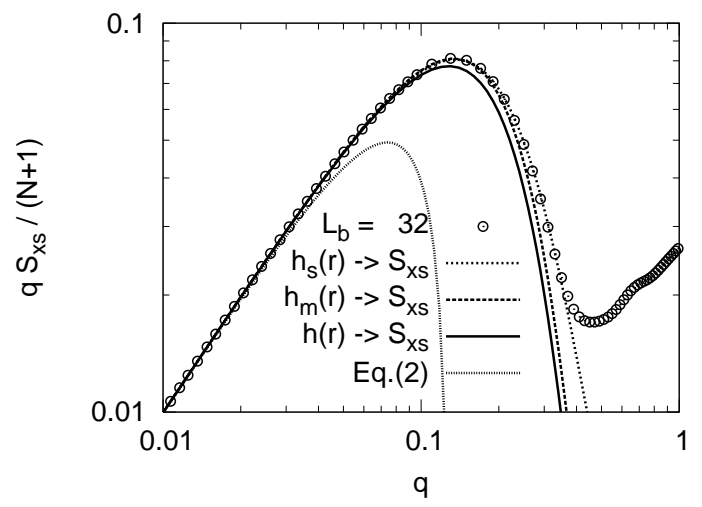

FIG. 14: Same as Fig. 13 but the fit functions are $h(r)$ and $h_{s}(r)$. Furthermore another fit is included which is extended up to the maximum in part (b) of this figure, $h_{m}(r)$.

the analysis using Eq. (11) may be warranted. This range extends at most to the position of the maximum in the plot of $q S_{\mathrm{xs}}(q)$ vs. $q$. In this q-range, one should employ a concave fitting function like the empirical law given by the function $h(r)$ above and not the convex forms usually assumed for the inner part of the brushes. The grafting densities typically employed in experiment are not high enough to lead to a radial density which resembles a filled cylinder with a smeared out interface to the solution when one works at good solvent or $\Theta$-solvent conditions. This assumption may be valid working in poor solvent, a regime which was not accessible to us using our simulation approach. When we perform a fit to the cross sectional scattering only for momentum transfers smaller than the peak position in the plots of $q S_{\mathrm{xs}}(q)$ vs. $q$, we obtain the function $h_{m}(r)$ included in Fig. 14. We can see that this is a good representation of the radial density down to values of about $\rho=0.01$.

As a final result let us discuss the cross sectional radius of gyration of the brush defined by

$$
R_{g c}^{2}=\frac{\int_{0}^{\infty} \rho(r) r^{2} 2 \pi r d r}{\int_{0}^{\infty} \rho(r) 2 \pi r d r} .
$$

Table 1 gives the resulting radii of gyration for the different fitting functions and procedures employed and for 
(a)

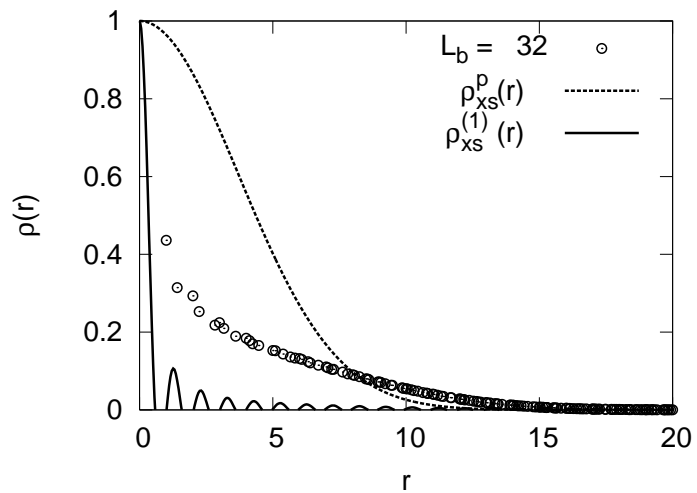

(b)

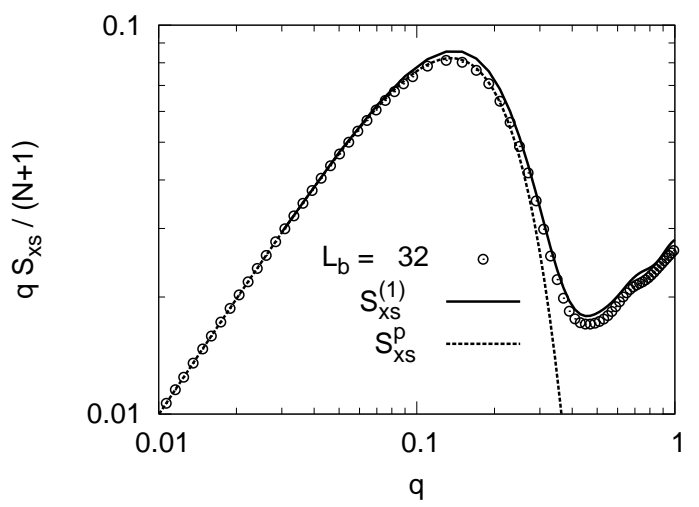

FIG. 15: (a) Monte Carlo data for the radial distribution function $\rho(r)$ of the monomers plotted versus $r$ for the side chain length $N=50, \sigma=1$ the good solvent case, and backbone length $L_{b}=32$. The full curve labeled $\rho_{\mathrm{xs}}^{(1)}(r)$ shows the result of Bessel transforming (Eq. (12)) the simulation data for the scattering function into real space. $\rho_{\mathrm{xs}}^{p}(r)$ shows the prediction for $\rho(r)$ obtained from fitting $s_{\mathrm{xs}}(q)$ using Eq. (14) (fit parameters are $R_{c}=1.0$, and $s=3.67$ ). (b) The cross sectional scattering $q S_{\mathrm{xs}}(q)$ is plotted vs. $q$. Symbols are data points, $S_{\mathrm{xs}}^{(1)}$ (full line) is the Bessel transform of the full curve in part (a) which should ideally coincide with the symbols (see text). $S_{\mathrm{xs}}^{p}$ (dashed line) is the best fit of Eq. (14) to the data.

TABLE I: Results for the cross sectional radius of gyration (see text) for the different fitting procedures and both solvent conditions.

\begin{tabular}{|l|lll|ll|l|}
\hline & $h(r)$ & $h_{m}(r)$ & $h_{s}(r)$ & $g(r)$ & $g_{s}(r)$ & $\rho_{\mathrm{xs}}^{p}(r)$ \\
\hline$R_{g c}$ (good solvent) & 7.83 & 7.49 & 7.64 & 7.94 & 7.79 & 5.24 \\
$R_{g c}(\Theta$-solvent) & 6.19 & 5.87 & 5.98 & 6.39 & 6.27 & 4.08 \\
\hline
\end{tabular}

the cases of good solvent and $\Theta$-solvent. All fitting procedures reproduce the shrinking of the brush going from good solvent to $\Theta$-solvent condition. The results using the functional forms $h(r)$ and $g(r)$ agree well with each other and also the suggested fit analysis of the scattering yielding function $h_{m}(r)$ results in only $4 \%$ deviation from the true value. The fits using Eq. (14), however, underestimate $R_{g c}$ by about $33 \%$. (a)

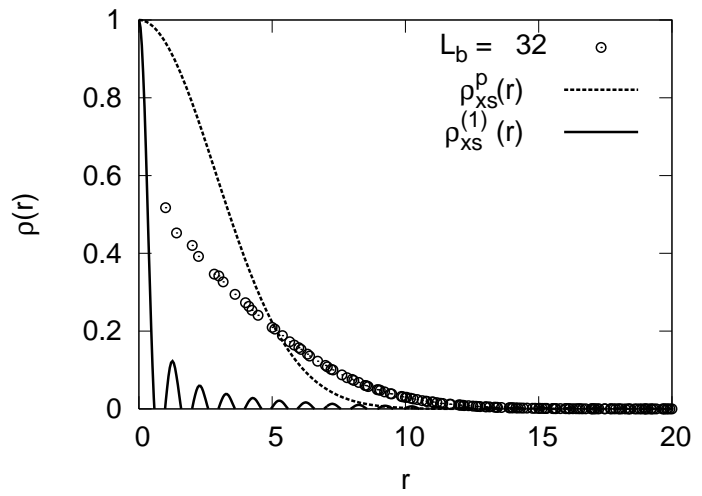

(b)

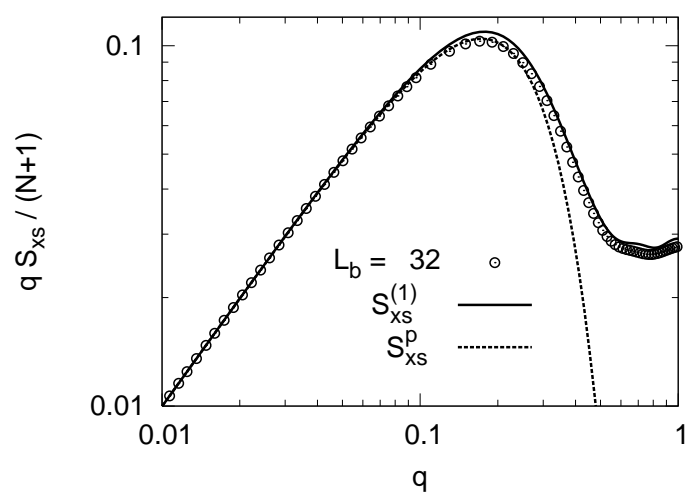

FIG. 16: Same as Fig. 15 for $\Theta$-solvent conditions. (fit parameters are $R_{c}=1.0$, and $s=2.88$ )

\section{CONCLUDING REMARKS}

In this paper, a comparative Monte Carlo study of bottle-brush polymers with rigid and relatively long backbone lengths ( $L_{b}=32$ to $L_{b}=256$ monomeric units) and flexible side chains of medium length (up to $N=50$ monomeric units) under good solvent and $\Theta$-solvent conditions was performed, using the PERM algorithm. The purpose of this study was to investigate the structure of such macromolecules and to test physical assumptions used in experimental work on related systems to extract structural information from scattering data.

Our main results can be summarized as follows:

(i) For the chosen side chain lengths, the chosen backbone lengths already are clearly outside of the crossover regime from bottle-brush to star polymer behavior. Comparing the total scattering function $S_{\mathrm{w}}(q)$ of a bottle-brush polymer with and without pbcs along the backbone, one does not find any pronounced effect due to the different conformations the chains at the end can assume in the two cases (therefore Fig. 9 only shows the scattering for the free boundary case). In addition, the range along the backbone over which the effect of the proximity of the free end of the backbone is felt in the side 
chain conformations is a few monomer diameters only.

(ii) Corroborating our earlier results 34] we find scaling concepts in terms of power laws, blob pictures etc. not useful to understand our results. We believe that scaling will become useful if the chain lengths of the side chains are two orders of magnitude larger; however, this limiting case is beyond the reach of either experiment or simulation.

(iii) Correlations between backbone monomers and side chain monomers do not contribute significantly to the scattering, while correlations between monomers from side chains anchoring at different backbone positions do. As a consequence, the standard factorization approximation by which the cross-sectional scattering function $S_{\mathrm{xs}}(q)$ is related via Fourier transform to the radial monomer density profile $\rho(r)$, is invalid for most of the momentum transfer range typically studied. While experiments typically are done for bottle-brush polymers with flexible backbones and we deal here with the case of rigid backbones only, there is no reason why approximations that are inaccurate in the latter case should become accurate in the flexible backbone case, of course. From a detailed analysis of the scattering function and radial density obtained in the simulation we identify the regime where the analysis of the cross sectional scattering might be successfully performed to lie at q-values smaller than the position of the peak in the curves of plots of $q S_{\mathrm{xs}}(q)$ vs. $q$. Here one should fit the Fourier transform of a concave form of radial density dependence, as given, e.g., by Eq. (16).

(iv) It would be desirable to perform neutron scattering from bottle brushes where only a small fraction of side chains is deuterated. In this way, a more direct information on the local conformational structure in a bottle-brush could be gained, and more extensive comparison with simulations should become possible. We also hope that our study will stimulate further experimental work on bottle brushes, in particular on the effect of solvent quality.

Acknowledgments: One of us (H.-P. H.) received financial support from the Deutsche Forschungsgemeinschaft (DFG) via Sonderforschungsbereich SFB 625/A3. We are grateful to S. Rathgeber and M. Schmidt for many stimulating discussions.
[1] M. Wintermantel, M. Schmidt, Y. Tsukahara, K. Kajiwara, and S. Koljiya, Macromol. Rapid Comm. 15, 279 (1994).

[2] M. Zhang and A. H. E. Müller, J. Polym. Sci., Part A: Polym. Chem. 43, 3461 (2005).

[3] S. Rathgeber, T. Pakula, A. Wilk, K. Matyjaszewski, and K. L. Beers, J. Chem. Phys. 122, 124904 (2005).

[4] S. Rathgeber, T. Pakula, A. Wilk, K. Matyjaswezski, H.I. Lee, and K. L. Beers, Polymer 47, 7318 (2006).

[5] B. Zhang, F. Gröhn, J. S. Pedersen, K. Fischer, and M. Schmidt, Macromolecules 39, 8440 (2006).

[6] L. Feuz, P. Strunz, T. Geue, M. Textor, and O. Borisov, Eur. Phys. J. E 23, 237 (2007).

[7] S. Bolisetty, C. Airaud, Y. Xu, A. H. E. Müller, L. Harnau, S. Rosenfeldt, P. Lindner, and M. Ballauff, Phys. Rev. E 75, 040803 (R) (2007).

[8] T. M. Birshtein and E. B. Zhulina, Polymer 25, 1453 (1984).

[9] T. A. Witten and P. A. Pincus, Macromolecules 19, 2509 (1986).

[10] T. M. Birshtein, O. V. Borisov, E. B. Zhulina, A. R. Khokhlov, and T. A. Yurasowa, Polym. Sci. USSR 29, 1293 (1987).

[11] Z.-G. Wang and S. A. Safran, J. Chem. Phys. 89, 5323 (1988).

[12] C. Ligoure and L. Leibler, Macromolecules 23, 5044 (1990).

[13] R. C. Ball, J. F. Marko, S. T. Milner, and T. A. Witten, Macromolecules 24, 693 (1991).

[14] M. Murat and G. S. Grest, Macromolecues 24, 704 (1991).

[15] N. Dan and M. Tirrell, Macromolecuels 25, 2890 (1992).
[16] C. M. Wijmans and E. B. Zhulina, Macromolecules 26, 7214 (1993).

[17] G. H,. Fredrickson, Macromolecules 26, 2825 (1993).

[18] E. B. Zhulina and T. A. Vilgis, Macromolecules 28, 1008 (1995).

[19] E. M. Sevick, Macromolecules 29, 6952 (1996).

[20] Y. Rouault and O. V. Borisov, Macromolecules 29, 2605 (1996).

[21] M. Saariaho, O. Ikkela, I. Szleifer, I. Erukhimovich, and G. ten Brinke, J. Chem. Phys. 107, 3267 (1997).

[22] M. Saariaho, I. Szleifer, O. Ikkala, and G. ten Brinke, Macromol. Theory Simul. 7, 211 (1998).

[23] Y. Rouault, Macromol. Theory Simul. 7, 359 (1989).

[24] M. Saariaho, A. Subbotin, I. Szleifer, O. Ikkala, and G. ten Brinke, Macromolecules 32, 4439 (1999).

[25] K. Shiokawa, K. Itoh, and M. Nemoto, J. Chem. Phys. 111, 8165 (1999).

[26] A. Subbotin, M. Saariaho, O. Ikkala, and G. ten Brinke, Macromolecules 33, 3447 (2000).

[27] P. G. Khalatur, D. G. Shirvanyanz, N. Y. Starovoitova, and A. R. Khokhlov, Macromol. Theory Simul. 9, 141 (2000).

[28] V. V. Vasilevskaya, A. A. Klochkov, R. G. Khalatur, A. R. Khokhlov, and G. ten Brinke, Macromol. Theory Simul. 10, 389 (2001).

[29] N. A. Denesyuk, Phys. Rev. E67, 051803 (2003).

[30] S. S. Sheiko, O. V. Borisov, S. A. Prokhorova and M. Möller, Eur. Phys. J. E13, 125 (2004).

[31] S. Elli, F. Ganazzoli, E. G. Timoshenko, Y. A. Kuznetsov, and R. Connolly, J. Chem. Phys. 120, 6257 (2004).

[32] R. Connolly, G. Bellesia, E. G. Timoshenko, Y. A. 
Kuznetsov, S. Elli, and F. Ganazzoli, Macromolecules 38, 5288 (2005).

[33] A. Yethiraj, J. Chem. Phys. 125, 204901 (2006).

[34] H.-P. Hsu, W. Paul, and K. Binder, Macromol. Theory Simul. 16, 660 (2007).

[35] H.-P. Hsu, W. Paul, and K. Binder, Macromol. Symp. 252, 58 (2007).

[36] C. Li, N. Gunari, K. Fischer, A. Janshoff, and M. Schmidt, Angew. Chem. Int. Ed. 43, 1101 (2004).

[37] N. Gunari, M. Schmidt, and A. Janshoff, Macromolecules 39, 2219 (2006).

[38] P. Grassberger, Phys. Rev. E56, 3682 (1997).

[39] P. G. de Gennes, Scaling Concepts in Polymer Physics
(Cornell Unvierstiy Press, Ithaca, New York, 1979).

[40] K. Kremer and K. Binder, Computer Phys. Repts. 7, 259 (1988).

[41] A. D. Sokal, in Monte Carlo and Molecular Dynamics Simulations in Polymer Science, edited by K. Binder (Oxford Univ. Press, New York 1995) p. 47.

[42] J. S. Pedersen and P. Schurtenberger, Macromolecules 29, 7602 (1996).

[43] T. Neugebauer, Ann. Phys. 434, 509 (1943).

[44] W. H. Press, S. A. Teukolsky, W. T. Vetterling and B. P. Flannery, Numerical Recipes. The Art of Scientific Computing (Cambridge Univ. Press, Cambridge, 2007) 